\title{
Radiation Reaction in Schwarzschild Spacetime: Retarded Green's Function via Hadamard-WKB Expansion
}

\author{
Paul R. Anderson ${ }^{1}$ and B. L. Hu ${ }^{2}$ \\ ${ }^{1}$ Department of Physics, Wake Forest University, Winston-Salem, NC 27109, USA \\ ${ }^{2}$ Department of Physics, University of Maryland, College Park, MD 20742, USA
}

An analytic method is given for deriving the part of the retarded Green's function $v\left(x, x^{\prime}\right)$ that contributes to the tail term in the radiation reaction force felt by a particle coupled to a massless minimally coupled scalar field. The method gives an expansion of $v\left(x, x^{\prime}\right)$ for small separations of the points $x, x^{\prime}$ valid for an arbitrary static spherically symmetric spacetime. It is obtained by using a WKB approximation for the Euclidean Green's function for the massless minimally coupled scalar field and is equivalent to the DeWitt-Schwinger expansion for $v\left(x, x^{\prime}\right)$. The first few terms in this expansion are displayed here for the case of Schwarzschild spacetime.

\section{INTRODUCTION}

This latest version has two new appendices $A$ and $B$ containing respectively the two Errata in Ref. [53] and [54] to the version published in Physical Review D [52]. In this version we have made the corrections indicated therein.

The past several years have seen a resurgence of interest in the gravitational radiation reaction problem in part because space based gravitational wave detectors such as the Lasar Interferometer Space Antenna (LISA) are expected to be able to detect the gravitational radiation emitted when a compact object such as a stellar mass black hole or neutron star spirals into a supermassive black hole[1, 2]. In such a situation the change in the spacetime geometry of the supermassive black hole due to the presence of the compact object and the gravitational radiation it emits is negligible (except near the compact object) so it is sufficient to compute the trajectory of the compact object in the background geometry of the supermassive black hole[2]. The radiation reaction under these simplifying conditions is known as the "self-force".

The radiation reaction problem for a point charge radiating electromagnetic waves in a curved space background was first investigated by DeWitt and Brehme [3] and later by 
Hobbs [4]. Gravitational radiation reaction for a moving particle was considered by Mino et al [5] and Quinn and Wald [6]. The self-force on a particle interacting with a massless minimally coupled scalar field was considered by Quinn [7]. For a review see Ref. [8]. The scalar field case is of interest primarily because it contains many of the same features as the gravitational and electromagnetic cases while being much easier to work with. As found originally by DeWitt and Brehme [3] for the electromagnetic case, it is always possible to break the radiation reaction force into two parts, one that is local and which reduces to the standard Abraham-Lorentz-Dirac expression in the flat space limit, the other part which is nonlocal and consists of an integral of the retarded Green's function over the past trajectory of the particle [5, 6, 7]. The nonlocal part is often called the "tail" term.

Much effort has gone into the computation of the self-force in Schwarzschild and Kerr spacetimes [9, 10, 11, 12, 13, 14, 15, 16, 17, 18, 19, 20, 21, 22, 23, 24, 25, 26, 27, 28, 29]. In most schemes that do not involve the weak field limit it must be computed numerically by expanding either the retarded Green's function, or the field itself, in terms of spherical harmonics in Schwarzschild or the spin-weighted spheroidal harmonics in Kerr [13, 14, 15, 16, 17, 18, 19, 22, 24, 25, 26, 27, 28, 29]. However, a drawback of these methods is that it has not been possible to separate out the local and nonlocal parts. The result is that there are divergences which must be regularized. The usual way to treat these divergences is through subtractions that occur at the level of the modes, resulting in a finite mode sum. This is similar to adiabatic regularization [30, 31, 32, 33, 34] introduced in the context of quantum field theory in curved spacetime [35, 36]. Zeta function regularization has also been used [17]

There is another way to obtain the tail term, at least in principle, by computing the part of the retarded Green's function that contributes to it. In this paper we propose to use the Hadamard expansion of the retarded Green's function for this purpose. It is known that this expansion is valid only when the points are close together. The self force on the object at a particular spacetime point has contributions from all points over its past trajectory. In nonlocal processes it is not unreasonable to start by taking into account contributions from points closest to the object as they usually give a greater weight than those farther away. A quasilocal expansion such as the one we are suggesting is the logical way to start an analytic approximation. Even if it turns out not to capture the dominant contribution, it is still worthwhile to investigate its range of validity for the specific task at hand. If any such analytic approximation produces even marginally reasonable results, it can provide a relatively quick way of estimating the self-force for a given trajectory. If the results are 
accurate enough then it can be used in place of brute force numerical integrations, thus giving an immense economy of effort.

For a massless minimally coupled scalar field, the retarded Green's function takes the Hadamard form [7, 37, 38, 39] ${ }^{1}$

$$
G_{r e t}\left(x, x^{\prime}\right)=\theta\left(x, x^{\prime}\right)\left\{\frac{u\left(x, x^{\prime}\right)}{4 \pi} \delta\left[\sigma\left(x, x^{\prime}\right)\right]-\frac{v\left(x, x^{\prime}\right)}{8 \pi} \theta\left[-\sigma\left(x, x^{\prime}\right)\right]\right\}
$$

Here $\theta\left(x, x^{\prime}\right)$ is defined to be zero outside of the past light cone and one inside of it. The quantity $\sigma\left(x, x^{\prime}\right)$ is equal to one-half the square of the proper distance between the points $x$ and $x^{\prime}$ along the geodesic connecting them. The function $v\left(x, x^{\prime}\right)$ contributes to the tail part of the self-force. It obeys the equation

$$
\square_{x} v\left(x, x^{\prime}\right)=0
$$

Note that $v\left(x, x^{\prime}\right)$ is finite when the points come together.

For small separations of the points the functions $u$ and $v$ can be computed by using the DeWitt-Schwinger expansion [35, 40, 41, 42]. To second order in derivatives of the metric the result for $v$ is

$$
v\left(x, x^{\prime}\right)=-\frac{1}{6} R(x)
$$

Christensen's expansions [41] can be used to compute $v\left(x, x^{\prime}\right)$ to fourth order. Phillips and $\mathrm{Hu}$ [39] have computed it to sixth order for a conformally coupled massless scalar field. Their result is useful for the study of a massless minimally coupled field in Schwarzschild spacetime because in any spacetime with zero scalar curvature the Green's functions for the massless scalar field do not depend upon the coupling to the scalar curvature. However, taking the general expressions for the terms in an arbitrary spacetime from Ref. [39] and evaluating them for the Schwarzschild geometry is still nontrivial.

The results of these calculations show that for Schwarzschild spacetime the sixth order contribution to $v\left(x, x^{\prime}\right)$ from the DeWitt-Schwinger expansion gives the first nonvanishing term, and that is the only term which the results from Ref. [39] can provide. To compute the self-force more terms are needed from an expansion for $v\left(x, x^{\prime}\right)$. Also, given the fact

\footnotetext{
${ }^{1}$ Our definitions for the Hadamard expansion are equivalent to those of Ref. [37, 38, 39]. The conventions of Ref. [7] differ from ours. To obtain the convention for $G_{\text {ret }}$ in Ref. [7] let $G_{\text {ret }} \rightarrow G_{\text {ret }} /(4 \pi)$. Substituting this into Eq. (A1) and comparing with Eq. (9) of Ref. 7] gives $u\left(x, x^{\prime}\right)=U\left(x, x^{\prime}\right)$, and $v\left(x, x^{\prime}\right)=2 V\left(x, x^{\prime}\right)$.
} 
that no explicit computations of $v\left(x, x^{\prime}\right)$ have been done for Schwarzschild spacetime using the DeWitt-Schwinger expansion, it is of some general interest to obtain the first few nonvanishing terms in the series.

In this paper we describe a method we have developed to compute an expansion for $v\left(x, x^{\prime}\right)$ for a massless minimally coupled scalar field in a general static spherically symmetric spacetime. The method involves the use of a WKB expansion for the radial mode functions of the Euclidean Green's function for the scalar field. It gives the same results as the DeWittSchwinger expansion. Because of its reliance on a WKB expansion for the radial modes of the Euclidean Green's function, it should be straight-forward to generalize the method to the cases of the electromagnetic and gravitational fields in static spherically symmetric spacetimes. Since the scalar, electromagnetic, and gravitational wave equations in Kerr spacetime are separable [43, 44, 45], it is likely that the method can be adapted to those cases as well.

In Sec. II we show the relationship between the Euclidean Green's function and $v\left(x, x^{\prime}\right)$ for the massless minimally coupled scalar field in a general static spherically symmetric spacetime. In Section III our method of deriving an expansion for $v\left(x, x^{\prime}\right)$ in powers of $\left(x-x^{\prime}\right)$ is given and the first few terms of the expansion for Schwarzschild spacetime are displayed. We draw our conclusions in Section IV.

\section{EUCLIDEAN GREEN'S FUNCTION AND THE TAIL TERM}

The metric for a static spherically symmetric spacetime can be written in the form

$$
d s^{2}=-f(r) d t^{2}+h(r) d r^{2}+r^{2} d \Omega^{2} .
$$

where $f(r)$ and $h(r)$ are arbitrary functions of the radial coordinate and $d \Omega^{2}$ is the metric of a 2-sphere. Changing the time variable $t$ to $\tau=i t$ gives the Euclidean metric. (Quantities defined in the Euclidean space carry a subscript E). The Euclidean Green's function is a solution to the equation

$$
\square_{x} G_{E}\left(x, x^{\prime}\right)=-\frac{\delta\left(x, x^{\prime}\right)}{\sqrt{g_{E}}}
$$

where $g_{E}$ is the determinant of the Euclidean metric. If the analytic continuation

$$
\left(\tau-\tau^{\prime}\right)^{2} \rightarrow-\left(t-t^{\prime}\right)^{2}+i \epsilon
$$

is used with $\epsilon$ an infinitesimal positive real quantity then [46]

$$
G_{E}\left(-i \tau, \vec{x} ;-i \tau^{\prime}, \overrightarrow{x^{\prime}}\right)=i G_{F}\left(x, x^{\prime}\right)
$$


with $G_{F}$ the Feynman Green's function. Since [41]

$$
\operatorname{Im} G_{F}\left(x, x^{\prime}\right)=-\frac{1}{2} G^{(1)}\left(x, x^{\prime}\right)
$$

where $G^{(1)}\left(x, x^{\prime}\right)$ is the Hadamard Green function, it is clear that

$$
\operatorname{Re} G_{E}\left(-i \tau, \vec{x} ;-i \tau^{\prime}, \overrightarrow{x^{\prime}}\right)=\frac{1}{2} G^{(1)}\left(x, x^{\prime}\right)=\frac{u\left(x, x^{\prime}\right)}{8 \pi^{2} \sigma\left(x, x^{\prime}\right)}+\frac{v\left(x, x^{\prime}\right)}{16 \pi^{2}} \log \left[\left|\sigma\left(x, x^{\prime}\right)\right|\right]+\frac{w\left(x, x^{\prime}\right)}{16 \pi^{2}}
$$

where in the second equality we have expressed $G^{(1)}\left(x, x^{\prime}\right)$ in the Hadamard form [37, 38, 39].

Note that if the points are separated in the time direction, or if they are farther apart in the time direction than in other directions then [42, 46]

$$
\sigma\left(x, x^{\prime}\right)=-\frac{1}{2} f(r)\left(t-t^{\prime}\right)^{2}+O\left[\left(x-x^{\prime}\right)^{3}\right] .
$$

Thus $v\left(x, x^{\prime}\right)$ is proportional to the coefficient of the $\log \left[\left(\tau-\tau^{\prime}\right)^{2}\right]$ part of $G_{E}\left(x, x^{\prime}\right)$. It is this fact that will enable us to derive an expansion for $v$ by finding one for $G_{E}$ using a WKB approximation.

To find such an expansion for $G_{E}$ first note that in a spacetime with metric (2.1) an exact expression for $G_{E}$ is [47]

$$
G_{E}\left(x, x^{\prime}\right)=\frac{1}{4 \pi^{2}} \int_{0}^{\infty} d \omega \cos \left[\omega\left(\tau-\tau^{\prime}\right)\right] \sum_{\ell=0}^{\infty}(2 \ell+1) P_{\ell}(\cos \gamma) C_{\omega \ell} p_{\omega \ell}\left(r_{<}\right) q_{\omega \ell}\left(r_{>}\right) .
$$

Here $P_{\ell}$ is the Legendre Polynomial of the $\ell$ th order and $\cos \gamma \equiv \hat{\mathbf{x}} \cdot \hat{\mathbf{x}}^{\prime}$ is the direction cosine between the spatial vectors defining the points $x, x^{\prime}$. The notation $r_{>}\left(r_{<}\right)$refers to the larger (smaller) of $r$ and $r^{\prime}$. The mode function $p_{\omega \ell}$ satisfies the appropriate boundary condition at some small value of $r$ and $q_{\omega \ell}$ satisfies the appropriate boundary condition at some large value of $r$. They are both solutions to the radial equation

$$
\frac{1}{h} \frac{d^{2} S}{d r^{2}}+\left[\frac{2}{r h}+\frac{1}{2 f h} \frac{d f}{d r}-\frac{1}{2 h^{2}} \frac{d h}{d r}\right] \frac{d S}{d r}-\left[\frac{\omega^{2}}{f}+\frac{\ell(\ell+1)}{r^{2}}\right] S=0 .
$$

They satisfy the Wronskian condition

$$
C_{\omega \ell}\left[p_{\omega \ell} \frac{d q_{\omega \ell}}{d r}-q_{\omega \ell} \frac{d p_{\omega \ell}}{d r}\right]=-\frac{1}{r^{2}}\left(\frac{h}{f}\right)^{1 / 2} .
$$

A WKB approximation for the modes can be derived as follows: First define

$$
\begin{aligned}
& p_{\omega \ell}=\frac{1}{\left(2 r^{2} W\right)^{1 / 2}} \exp \left[\int^{r} W\left(\frac{h}{f}\right)^{1 / 2} d r\right], \\
& q_{\omega \ell}=\frac{1}{\left(2 r^{2} W\right)^{1 / 2}} \exp \left\{-\left[\int^{r} W\left(\frac{h}{f}\right)^{1 / 2} d r\right]\right\}
\end{aligned}
$$


with

$$
\begin{aligned}
W^{2} & =\Omega^{2}+\frac{1}{2 r h} \frac{d f}{d r}-\frac{f}{2 r h^{2}} \frac{d h}{d r} \\
& +\frac{1}{2}\left[\frac{f}{h W} \frac{d^{2} W}{d r^{2}}+\left(\frac{1}{h} \frac{d f}{d r}-\frac{f}{h^{2}} \frac{d h}{d r}\right) \frac{1}{2 W} \frac{d W}{d r}-\frac{3}{2} \frac{f}{h}\left(\frac{1}{W} \frac{d W}{d r}\right)^{2}\right]
\end{aligned}
$$

and

$$
\Omega^{2}=\omega^{2}+\ell(\ell+1) \frac{f}{r^{2}}
$$

Then solve the equation for $W$ iteratively, the lowest order solution being $W=\Omega$. Each iteration adds two derivatives of the metric. To second order one finds

$$
\begin{aligned}
W=\Omega & +\frac{1}{2 \Omega}\left(\frac{1}{2 r h} \frac{d f}{d r}-\frac{f}{2 r h^{2}} \frac{d h}{d r}\right)-\frac{1}{8 \Omega^{3}}\left(\frac{1}{2 r h} \frac{d f}{d r}-\frac{f}{2 r h^{2}} \frac{d h}{d r}\right)^{2} \\
& +\frac{1}{4}\left[\frac{f}{h \Omega^{2}} \frac{d^{2} \Omega}{d r^{2}}+\left(\frac{1}{h} \frac{d f}{d r}-\frac{f}{h^{2}} \frac{d h}{d r}\right) \frac{1}{2 \Omega^{2}} \frac{d \Omega}{d r}-\frac{3}{2} \frac{f}{h} \frac{1}{\Omega^{3}}\left(\frac{d \Omega}{d r}\right)^{2}\right]
\end{aligned}
$$

Substitution of Eq. (2.11) into Eq. (2.10) shows that for the WKB ansatz, $C_{\omega \ell}=1$.

\section{COMPUTATION OF $v\left(x, x^{\prime}\right)$}

To compute an expansion for $v\left(x, x^{\prime}\right)$ in powers of $\left(x-x^{\prime}\right)$ one first solves Eq.(2.12) to a specified adiabatic order and substitutes the result into Eq. (2.8). As shown below, the resulting expression can be broken into sums and integrals of the form

$$
\left.S_{m n p}=\int_{0}^{\infty} d \omega \cos \omega\left(\tau-\tau^{\prime}\right)\right] \omega^{2 n} \sum_{\ell=0}^{\infty}(2 \ell+1) \frac{[l(l+1)]^{m}}{\Omega^{p}}
$$

with $m, n$, and $p$ integers.

Our goal is to determine the coefficient of the $\log \left(\tau-\tau^{\prime}\right)$ term since this is related to $v\left(x, x^{\prime}\right)$ through Eqs. (2.6) and (2.7). This can be accomplished by using the Plana sum formula [48]

$$
\sum_{n=N}^{\infty} F(n)=\frac{1}{2} F(N)+\int_{N}^{\infty} d n F(n)+i \int \frac{d t}{e^{2 \pi t}-1}[F(N+i t)-F(N-i t)]
$$

to compute the sum over $\ell$. There are superficial divergences in some of the sums over $\ell$ which can be isolated by expanding the summand for a given sum in inverse powers of $\ell$ and then truncating at order $1 / \ell$. If the remaining terms are subtracted from the summand then the result will be finite. Effectively this amounts to subtracting terms which are proportional 
to $\delta\left(\tau-\tau^{\prime}\right)$ and its derivatives. This procedure is discussed in Ref. [47]. After computing the sum over $\ell$ one can expand the result in inverse powers of $\omega$. Most of the expressions will be infrared divergent, however this is not important because we are only looking for the coefficient of the $\log \left(\tau-\tau^{\prime}\right)$ term. ${ }^{2}$ Thus it suffices to put in a lower limit cutoff $\lambda$ on the integral over $\omega$.

The integral over $\omega$ can be computed for each term of the series. For positive powers of $\omega$ there are no $\log \left(\tau-\tau^{\prime}\right)$ terms so these do not contribute to $v\left(x, x^{\prime}\right)$. For all negative powers of $\omega$ there will be $\log \left(\tau-\tau^{\prime}\right)$ terms. For $\omega^{-1}$ one finds

$$
\int_{\lambda}^{\infty} d \omega \cos \left[\omega\left(\tau-\tau^{\prime}\right)\right] \frac{1}{\omega}=-\operatorname{ci}\left(\lambda\left(\tau-\tau^{\prime}\right)\right)=-\log \left(\tau-\tau^{\prime}\right)+\ldots .
$$

For all negative powers of $\omega$, successive integrations by parts can be performed until the integral is in the form (3.3). The result is

$$
\int d \omega \cos \left[\omega\left(\tau-\tau^{\prime}\right)\right] \frac{1}{\omega^{2 n+1}}=\frac{(-1)^{n+1}}{(2 n) !}\left(\tau-\tau^{\prime}\right)^{2 n} \log \left(\tau-\tau^{\prime}\right)+\ldots
$$

If the points are split only in the time direction then one finds that using a second order WKB expansion gives $v$ to zeroth order in $\left(t-t^{\prime}\right)$, a fourth order one gives $v$ to second order in $\left(t-t^{\prime}\right)$, and so forth. For Schwarzschild spacetime the lowest nonvanishing order is $\left(t-t^{\prime}\right)^{4}$ which requires use of a sixth order WKB approximation.

To compute the expansion when the points are also split in the angular direction one can begin by expanding the angular part of the mode functions in powers of $(\cos \gamma-1)$ so that

$$
P_{\ell}(\cos \gamma)=1+\frac{\ell(\ell+1)}{2}(\cos \gamma-1)+\ldots
$$

Then the sum over $\ell$ and the integral over $\omega$ can be computed as before. At the end of the calculation one should also expand the terms of the form $(\cos \gamma-1)^{j}$ in powers of $\theta-\theta^{\prime}$ and $\phi-\phi^{\prime}$

To compute the expansion when the points are also split in the radial direction one should first fix $r^{\prime}$ to be either less than or greater than $r$. For our purpose it will not matter which is chosen so, as an example, we assume that $r^{\prime}<r$. Then one expands $p_{\omega \ell}\left(r^{\prime}\right)$ in powers of $r^{\prime}-r$ and repeatedly uses the mode equation (2.9) to eliminate all but first derivatives of $p_{\omega \ell}$. Thus

$$
p_{\omega \ell}\left(r^{\prime}\right)=p_{\omega \ell}(r)+p_{\omega \ell}^{\prime}(r)\left(r^{\prime}-r\right)
$$

\footnotetext{
${ }^{2}$ There is an alternative way to do the WKB expansion which gives no infrared divergences [47, 49, 50].
} 


$$
+\left\{\left[-\frac{2}{r}-\frac{1}{2 f} \frac{d f}{d r}+\frac{1}{2 h} \frac{d h}{d r}\right] p_{\omega \ell}^{\prime}(r)+\left[\frac{\omega^{2} h}{f}+\frac{\ell(\ell+1) h}{r^{2}}\right] p_{\omega \ell}(r)\right\} \frac{\left(r^{\prime}-r\right)^{2}}{2}+\ldots
$$

Then this expansion is substituted into the expression (2.8) for $G_{E}$ and the WKB expansion is introduced to write this equation in terms of $W$ and its derivatives as before. The terms will be proportional to various powers of $\omega$ and $\ell(\ell+1)$ multiplying either $1,1 / W$, or $W^{\prime} / W^{2}$. Those multiplying 1 are proportional to $\delta\left(\tau-\tau^{\prime}\right) \delta\left(\Omega-\Omega^{\prime}\right)$ and various derivatives of these delta functions. Therefore, they do not contribute to the calculation of $v\left(x, x^{\prime}\right)$ and can be ignored. For the other terms one substitutes the WKB approximation for $W$ to some specified order and computes the coefficients of the $\log \left(\tau-\tau^{\prime}\right)$ terms as before.

To affirm the validity of the WKB scheme, we note that when this program is carried out for a Reissner-Nordström spacetime the expansion for $v\left(x, x^{\prime}\right)$ agrees with the results of Christensen [41] at order $\left(x-x^{\prime}\right)^{2}$. To leading order, it is also a solution to Eq. (1.21). For Schwarzschild spacetime $v\left(x, x^{\prime}\right)$ vanishes at order $\left(x-x^{\prime}\right)^{2}$. However, by using an eighth order WKB approximation for the modes it has been possible to compute $v\left(x, x^{\prime}\right)$ to order $\left(x-x^{\prime}\right)^{6}$ in Schwarzschild spacetime. Writing

$$
v\left(x, x^{\prime}\right)=\sum_{i, j, k=0}^{\infty} v_{i j k}\left(t-t^{\prime}\right)^{2 i}(\cos \gamma-1)^{j}\left(r-r^{\prime}\right)^{k}
$$

we find

$$
v_{000}=v_{001}=v_{100}=v_{010}=v_{002}=v_{101}=v_{011}=v_{003}=0
$$


and

$$
\begin{aligned}
& v_{200}=\frac{3 M^{2}(2 M-r)^{3}}{224 r^{11}} \\
& v_{110}=\frac{27 M^{2}(2 M-r)^{2}}{280 r^{8}} \\
& v_{102}=\frac{-9 M^{2}(2 M-r)}{560 r^{9}} \\
& v_{020}=\frac{9 M^{2}(2 M-r)}{280 r^{5}} \\
& v_{012}=\frac{-9 M^{2}}{280 r^{6}} \\
& v_{004}=\frac{3 M^{2}}{1120(2 M-r) r^{7}} \\
& v_{201}=\frac{3 M^{2}(11 M-4 r)(2 M-r)^{2}}{224 r^{12}} \\
& v_{111}=\frac{27 M^{2}(8 M-3 r)(2 M-r)}{280 r^{9}} \\
& v_{103}=\frac{-9 M^{2}(9 M-4 r)}{560 r^{10}} \\
& v_{021}=\frac{9 M^{2}(5 M-2 r)}{280 r^{6}} \\
& v_{013}=\frac{-27 M^{2}}{280 r^{7}} \\
& v_{005}=\frac{3 M^{2}(7 M-4 r)}{1120(2 M-r)^{2} r^{8}} \\
& v_{300}=\frac{M^{2}(2 M-r)^{3}\left(39 M^{2}-26 M r+4 r^{2}\right)}{480 r^{15}} \\
& v_{210}=\frac{M^{2}(2 M-r)^{2}\left(135 M^{2}-91 M r+14 r^{2}\right)}{224 r^{12}} \\
& v_{202}=\frac{M^{2}(2 M-r)\left(171 M^{2}-136 M r+26 r^{2}\right)}{224 r^{13}} \\
& v_{120}=\frac{9 M^{2}(2 M-r)\left(21 M^{2}-18 M r+4 r^{2}\right)}{560 r^{9}} \\
& v_{112}=\frac{3 M^{2}\left(567 M^{2}-473 M r+94 r^{2}\right)}{560 r^{10}} \\
& v_{104}=\frac{-3 M^{2}(51 M-28 r)(5 M-2 r)}{1120(2 M-r) r^{11}} \\
& v_{030}=\frac{M^{2}(3 M-14 r)(2 M-r)}{840 r^{6}} \\
& v_{022}=\frac{3 M^{2}\left(81 M^{2}-70 M r+14 r^{2}\right)}{560(2 M-r) r^{7}} \\
& v_{014}=\frac{-M^{2}\left(729 M^{2}-773 M r+202 r^{2}\right)}{1120(2 M-r)^{2} r^{8}} \\
& v_{006}=\frac{M^{2}\left(249 M^{2}-292 M r+86 r^{2}\right)}{3360(2 M-r)^{3} r^{9}}
\end{aligned}
$$


This expression is a solution to Eq. (1.2) to $O\left[\left(x-x^{\prime}\right)^{4}\right]$. By that we mean that if it is substituted into the left hand side of Eq. (1.2) then the resulting expression will be zero to $O\left[\left(x-x^{\prime}\right)^{4}\right]$.

\section{CONCLUSIONS}

We have presented a method that allows for the calculation of the part of the retarded Green's function $v\left(x, x^{\prime}\right)$ that contributes to the tail part of the radiation reaction force for a massless minimally coupled scalar field in a general static spherically symmetric spacetime. We expect it to be straight-forward to adapt this method to the cases of the electromagnetic and gravitational fields in static spherically symmetric spacetimes. It may be possible to adapt it, for all three fields, to Kerr spacetime as well given that the wave equations for all three fields are separable in the Kerr background [43, 44, 45].

We have explicitly calculated $v\left(x, x^{\prime}\right)$ in Schwarzschild spacetime to $O\left[\left(x-x^{\prime}\right)^{6}\right]$ for an arbitrary separation of the points. One indication of the correctness of these expressions is that $v\left(x, x^{\prime}\right)$ satisfies Eq. (1.2) to the appropriate order.

Although we have not yet computed enough terms to get an estimate of the self-force, one qualitative feature in our results is distinct from other related cases [51]. In the large $r$ limit one can see that the leading order terms in $v$ are all proportional to $M^{2}$. By comparison, calculations by DeWitt and DeWitt [9] and Pfenning and Poisson [23] show that when the spacetime curvature is everywhere small, such as is the case for a static star, then the leading order term is proportional to $M$. More specifically, in the calculation of DeWitt and DeWitt the metric is everywhere Schwarzshild except at the origin where they assume a delta function mass source. They made the approximation that the Green's function can be well approximated everywhere by the flat space Green's function (this is known to be false for regions close to the black hole event horizon)and found that the leading order term comes from a signal that propagates to the central condensation at $r=0$, bounces off and comes back to the current location of the particle.

One might be concerned that similar processes will contribute a leading order term that is linear in $M$ for the black hole case. This may well turn out to be the case, and one should consider this factor seriously. However, before drawing any direct implications we caution that the case of a particle orbiting a static star is qualitatively different from that of a particle orbiting a black hole. For example, the calculations in Ref. [9, 23] assume that 
the curvature is nowhere large which is not a valid assumption for the case of a particle orbiting a black hole. Also, in the case of a black hole the existence of an event horizon precludes classical waves from scattering off the central condensation (note this is different from the superradiance effect occurring in the ergosphere of a Kerr black hole), even though such scattering can occur for orbits with $r>3 M$ everywhere. We think it likely that this type of nonlocal (infrared) contribution to the self-force can be, at least approximately, decoupled from the quasilocal contribution we are considering here. If this is the case, then the nonlocal contribution can be considered separately and the result can be added to the quasilocal contribution to the self-force.

The method we have developed to compute $v\left(x, x^{\prime}\right)$ can be used to find the expansion to higher orders in $\left(x-x^{\prime}\right)$. Work is in progress to compute an expansion for $v\left(x, x^{\prime}\right)$ to substantially higher order in Schwarzschild spacetime, which should provide a better test of the usefulness of the Hadamard expansion. Although the quasilocal expansion may prove insufficient to compute the tail part of the self-force, the facts that no regularization is necessary and the resulting expression is analytic make an investigation of its usefulness well worth the effort. Such an investigation is underway and the results will be presented elsewhere.

\section{Acknowledgments}

We would like to thank Nicholas Phillips for helpful conversations, Payman Eftekharzadeh for detecting (non-propagating) errors in two equations of our original draft, and Eric Poisson

for pointing out the likely existence of a linear $M$ term in the expression for the self-force, which the present approximation lacks. This work was supported in part by the National Science Foundation under grant numbers PHY-0070981 and PHY-0300710.

[1] B. F. Schutz, Class.Quant.Grav. 16 A131-A156, (1999).

[2] S. A. Hughes, Annals Phys. 303, 142 (2003).

[3] B. S. DeWitt and R. W. Brehme, Ann. Phys. (N.Y.) 9, 220 (1960).

[4] J. M. Hobbs, Ann. Phys. (NY) 47, 141 (1968).

[5] Y. Mino, M. Sasaki, and T. Tanaka, Phys. Rev. D 55, 3457 (1997).

[6] T. C. Quinn and R. M. Wald, Phys. Rev. D 56, 3381 (1997). 
[7] T. C. Quinn, Phys. Rev. D 62, 064029 (2000).

[8] E. Poisson, Preprint: gr-qc/0306052.

[9] C. M. DeWitt and B. S. DeWitt, Physics 1, 3 (1964).

[10] C. H. MacGruder III, Nature (London) 272, 806 (1978).

[11] A. Vilenkin, Phys. Rev. D 20, 373 (1979).

[12] A. G. Smith and C. M. Will, Phys. Rev. D 22, 1276 (1980).

[13] A. Ori, Phys. Lett. A 202, 347 (1995).

[14] A. Ori, Phys. Rev. D 55, 3444 (1997).

[15] L. Barack and A. Ori, Phys. Rev. D 61, 061502(R) (2000).

[16] L. M. Burko, Class. Quantum Grav. 17, 227 (2000); Phys. Rev. Lett. 84, 4529 (2000).

[17] C. O. Lousto, Phys. Rev. Lett. 84, 5251 (2000).

[18] L. Barack, Phys. Rev. D 62, 084027 (2000);D 64084021 (2001).

[19] L. Barack and L. M. Burko, Phys. Rev. D 62, 084040 (2000).

[20] S. Detweiler, Phys. Rev. Lett. 86, 1931 (2001).

[21] H. Nakano, Y. Mino and M. Sasaki, Prog. Theor. Phys. 106339 (2001).

[22] L. M. Burko and Y. T. Liu, Phys. Rev. D 64024006 (2001).

[23] M. J. Pfenning and E. Poisson, Phys. Rev. D 65084001 (2002).

[24] L. Barack and C. O. Lousto, Phys. Rev. D 66061502 (2002).

[25] L. Barack, Y. Mino, H. Nakano, A. Ori, and M. Sasaki, Phys. Rev. Lett. 88091101 (2002).

[26] L. Barack and A. Ori, Phys.Rev. D66 (2002) 084022; Phys.Rev. D67, 024029 (2003); Phys. Rev. Lett. 90, 111101 (2003).

[27] Y. Mino, H. Nakano, M. Sasaki, Prog. Theor. Phys. 1081039 (2003).

[28] S. Detweiler and B. F. Whiting, Phys. Rev. D67 (2003) 024025.

[29] S. Detweiler, E. Messaritaki, and B. F. Whiting, Phys. Rev. D67 (2003) 104016.

[30] L. Parker, Ph.D. thesis, Harvard University, 1966 (Xerox University Microfilms, Ann Arbor, Michigan, No. 73-31244), pp. 140-171 and App. CI.

[31] L. Parker and S. A. Fulling. Phys. Rev. D 9, 341 (1974).

[32] S. A. Fulling and L. Parker. Ann. Phys. (N. Y.) 87, 176 (1974).

[33] B. L. Hu, Phys. Rev. D 9, 3263 (1974)

[34] S. A. Fulling, L. Parker, and B. L. Hu. Phys. Rev. D 10, 3905 (1974).

[35] B. S. DeWitt, in Relativity, Groups and Topology, edited by B. S. DeWitt and C. DeWitt (Gordon and Breach, New York, 1065); Phys. Rep. 19, 295 (1975). 
[36] N. D. Birrell and P. C. W. Davies, Quantum Fields in Curved Space, Cambridge University Press (Cambridge, 1982) and references therein.

[37] S. L. Adler, J. Lieberman and Y. J. Ng, Ann. Phys. (N.Y.) 106, 279 (1977).

[38] R. M. Wald, Commun. Math. Phys. 45, 9 (1975); Phys. Rev. D17, 1477 (1978).

[39] N. G. Phillips and B. L. Hu, Phys. Rev. D 63, 104001 (2001); 67, 104002 (2003).

[40] J. Schwinger, Phys. Rev. 82, 664 (1951).

[41] S. M. Christensen, Phys. Rev. D 14, 2490 (1976).

[42] S. M. Christensen, Ph.D. thesis, University of Texas at Austin, 1975.

[43] B. Carter, Commun. Math. Phys. 10, 280 (1968).

[44] D. R. Brill, P. L. Chrzanowski, C. M. Pereira, E. D. Fackerell, and J. R. Ipser, Phys. Rev. D 5, 1913 (1972).

[45] S. A. Teukolsky, Phys. Rev. Lett. 29, 1114 (1972).

[46] P. B. Groves, P. R. Anderson, and E. D. Carlson, Phys. Rev. D 66, 124017 (2002).

[47] P. R. Anderson, W. A. Hiscock, and D. A. Samuel, Phys. Rev. Lett. 70, 1739 (1993); Phys. Rev. D 51, 4337 (1995).

[48] E. T. Whitaker and G. N. Watson, A Course of Modern Analysis (Cambridge University Press, Cambridge, England, 1927), p. 145, exercise 7. G. Ghika and M. Visinescu, Nuovo Cimento A 46, 25 (1978).

[49] P. Candelas and K. W. Howard, Phys. Rev. D 29, 1618 (1984).

[50] K. W. Howard, Phys. Rev. D 30, 2532 (1984).

[51] E. Poisson, Private communication.

[52] P. R. Anderson and B. L. Hu, Phys. Rev. D69 064039 (2004).

[53] P. R. Anderson and B. L. Hu, Erratum, Phys. Rev. D75, 129901(E)(2007).

[54] P. R. Anderson and B. L. Hu, Erratum, Phys. Rev. D77, 089901 (E) (2008). 


\section{APPENDIX A: FIRST ERRATUM}

In the Physical Review D (PRD) version of this paper [52] there were a few notational and transcription errors that have been corrected in this arXiv version and also published in an erratum [53]. For completeness we list the corrections in that erratum here as well.

1. In Eq. (1.1) of the PRD version the general expression for the Hadamard form of the retarded Green's function was given incorrectly. It should read

$$
G_{\mathrm{ret}}\left(x, x^{\prime}\right)=\theta\left(x, x^{\prime}\right)\left\{\frac{u\left(x, x^{\prime}\right)}{4 \pi} \delta\left[\sigma\left(x, x^{\prime}\right)\right]-\frac{v\left(x, x^{\prime}\right)}{8 \pi} \theta\left[-\sigma\left(x, x^{\prime}\right)\right]\right\}
$$

2. The relationships between our definitions of $u\left(x, x^{\prime}\right)$ and $v\left(x, x^{\prime}\right)$ above and $U\left(x, x^{\prime}\right)$ and $V\left(x, x^{\prime}\right)$ of Ref. [7] were given incorrectly in the PRD version. To obtain the correct relationships first note that the convention for $G_{r e t}$ in Ref. [52] differs from that of Ref. [7]. To obtain that of Ref. [7] let $G_{\text {ret }} \rightarrow G_{\text {ret }} /(4 \pi)$. Substituting this into Eq. (A1) and comparing with Eq. (9) of Ref. [7] gives $u\left(x, x^{\prime}\right)=U\left(x, x^{\prime}\right)$, and $v\left(x, x^{\prime}\right)=2 V\left(x, x^{\prime}\right)$.

3. In Eq. (3.9) of the PRD version the expressions for the following quantities should be multiplied by a minus sign: $v_{201}, v_{111}, v_{103}, v_{021}, v_{013}$, and $v_{005}$.

These errors do not affect any other equations or results in the paper and the above corrections have been made in this arXiv version.

We would like to thank Ardeshir Eftekharzadeh (April 2006) for first pointing out the errors in Eq. (3.9) of the PRD version, and Barry Wardell (April 2007) for independently pointing them out. P. R. A. would like to thank Hebrew University and the Department of Theoretical Physics at the University of Valencia for hospitality. This work was supported in part by the National Science Foundation under grant numbers PHY03-00710 and PHY0556292. P.R.A. acknowledges the Einstein Center at Hebrew University, the Forchheimer Foundation, and the Spanish Ministerio de Educación y Ciencia for financial support. 


\section{APPENDIX B: SECOND ERRATUM}

In the Physical Review D (PRD) version of this paper [52] there was a factor of 2 error that has been corrected in this arXiv version and also published in an erratum [54]. For completeness we list the corrections in that erratum here as well.

In Ref. [52] there was an error in the calculation of the coefficients in the Hadamard-WKB expansion of $v\left(x, x^{\prime}\right)$. Each coefficient in Eq. (3.9) should be multiplied by a factor or 2. A transcriptional error in certain coefficients was corrected in [53]. The expansion for $v$ is

$$
v\left(x, x^{\prime}\right)=\sum_{i, j, k=0}^{\infty} v_{i j k}\left(t-t^{\prime}\right)^{2 i}(\cos \gamma-1)^{j}\left(r-r^{\prime}\right)^{k}
$$

The corrected coefficients, including both the corrections in [53] and the factor of 2, are

$$
v_{000}=v_{001}=v_{100}=v_{010}=v_{002}=v_{101}=v_{011}=v_{003}=0
$$


and

$$
\begin{aligned}
& v_{200}=\frac{3 M^{2}(2 M-r)^{3}}{224 r^{11}} \\
& v_{110}=\frac{27 M^{2}(2 M-r)^{2}}{280 r^{8}} \\
& v_{102}=\frac{-9 M^{2}(2 M-r)}{560 r^{9}} \\
& v_{020}=\frac{9 M^{2}(2 M-r)}{280 r^{5}} \\
& v_{012}=\frac{-9 M^{2}}{280 r^{6}} \\
& v_{004}=\frac{3 M^{2}}{1120(2 M-r) r^{7}} \\
& v_{201}=\frac{3 M^{2}(11 M-4 r)(2 M-r)^{2}}{224 r^{12}} \\
& v_{111}=\frac{27 M^{2}(8 M-3 r)(2 M-r)}{280 r^{9}} \\
& v_{103}=\frac{-9 M^{2}(9 M-4 r)}{560 r^{10}} \\
& v_{021}=\frac{9 M^{2}(5 M-2 r)}{280 r^{6}} \\
& v_{013}=\frac{-27 M^{2}}{280 r^{7}} \\
& v_{005}=\frac{3 M^{2}(7 M-4 r)}{1120(2 M-r)^{2} r^{8}} \\
& v_{300}=\frac{M^{2}(2 M-r)^{3}\left(39 M^{2}-26 M r+4 r^{2}\right)}{480 r^{15}} \\
& v_{210}=\frac{M^{2}(2 M-r)^{2}\left(135 M^{2}-91 M r+14 r^{2}\right)}{224 r^{12}} \\
& v_{202}=\frac{M^{2}(2 M-r)\left(171 M^{2}-136 M r+26 r^{2}\right)}{224 r^{13}} \\
& v_{120}=\frac{9 M^{2}(2 M-r)\left(21 M^{2}-18 M r+4 r^{2}\right)}{560 r^{9}} \\
& v_{112}=\frac{3 M^{2}\left(567 M^{2}-473 M r+94 r^{2}\right)}{560 r^{10}} \\
& v_{104}=\frac{-3 M^{2}(51 M-28 r)(5 M-2 r)}{1120(2 M-r) r^{11}} \\
& v_{030}=\frac{M^{2}(3 M-14 r)(2 M-r)}{840 r^{6}} \\
& v_{022}=\frac{3 M^{2}\left(81 M^{2}-70 M r+14 r^{2}\right)}{560(2 M-r) r^{7}} \\
& v_{014}=\frac{-M^{2}\left(729 M^{2}-773 M r+202 r^{2}\right)}{1120(2 M-r)^{2} r^{8}} \\
& v_{006}=\frac{M^{2}\left(249 M^{2}-292 M r+86 r^{2}\right)}{3360(2 M-r)^{3} r^{9}}
\end{aligned}
$$




\section{ACKNOWLEDGMENTS}

We would like to thank Barry Wardell for alerting us to the possibility that a factor of two error existed in our results and for help in finding it. We would also like thank Ardeshir Eftekharzadeh for help in finding the error. P. R. A. would like to thank the Physics Department at the University of Bologna for hospitality. This work was supported in part by the National Science Foundation under grant numbers PHY03-00710 and PHY05-56292. 